\title{
INTERNET PER LE PICCOLE E MEDIE IMPRESE: POTENZIALITÀ E STATO DELL'ARTE IN ITALIA E IN EUROPA
}

\author{
Elena Cedrola*
}

\begin{abstract}
Small and medium enterprises: state of the art an perspectives in Italy and Europe

The article focuses on the role attributed to the Internet by small and medium enterprises. In 2004, 2006, and 2007 the author investigated a sample of companies belonging to the main economic sectors. Regarding 2007, the watching also covered a sample of European companies in order to identify similarities and differences.

The empirical evidence lead to a scenario studded with lights and shadows. The lights concerns the improvements achieved, and are mainly related to the structure of web sites, relationship, and the presentation of products. The shadows relate to some elements of the Internet marketing mix (distribution, communication and pricing), and are also connected to the strategic guidelines of the enterprises.
\end{abstract}

Keywords: Internet, Internet marketing mix, small and medium enterprises, Italy, Europe, empirical research

\section{Riassunto}

L'articolo si focalizza sul ruolo attribuito ad internet da parte delle piccole e medie imprese. A questo scopo l'autore ha indagato, negli anni 2004, 2006 e 2007, un campione di aziende appartenenti ai principali settori economici. Per quanto concerne il 2007 il watching ha riguardato anche un campione di imprese europee, al fine di individuare similitudini e differenze di approccio.

Le evidenze empiriche conducono ad uno scenario costellato di luci ed ombre. Le luci sono relative ai numerosi miglioramenti intervenuti sui siti, che attengono principalmente alla struttura, alla relazione e alla presentazione dei prodotti. Le ombre invece, oltre a interessare gli altri elementi dell'internet marketing mix, riguardano lo scarso orientamento strategico delle aziende.

Parole chiave: internet, internet marketing mix, piccole e medie imprese, Italia, Europa, ricerca empirica.

* Professore Associato di Economia e Gestione delle Imprese presso l'Università di Macerata e docente incaricato di Marketing Internazionale presso l'Università Cattolica del Sacro Cuore di Milano. E-mail: elena.cedrola@unimc.it. 


\section{E. Cedrola}

\section{Internet e piccole e medie imprese}

La rilevanza del ruolo delle piccole e medie imprese (PMI) nella struttura industriale di numerosi paesi ha stimolato la ricerca a concentrare l'attenzione sulle dinamiche che caratterizzano la presenza in rete di questa tipologia d'impresa (MacGregor e Vrasalic, 2007 - Gilmore e Gallagher e Henry, 2007 Canavan et al., 2007 - Cioppi, 2006 - Kaynak e Tatoglu e Kula, 2005 - Stockdale e Standing, 2004 - Stone, 2003 - Poon e Swatman, 1997). Ciò nel tentativo di comprendere le logiche che guidano gli imprenditori ad investire nel web e, nel contempo, di identificare possibili percorsi di sviluppo che consentano lo sfruttamento delle potenzialità che internet è in grado di offrire alle PMI. Potenzialità concretizzabili sia in termini di abbattimento dei costi e delle barriere spazio-temporali, sia di incremento della competitività-cooperazione, sia di orientamento al cliente e di sviluppo delle relazioni di filiera e con il cliente finale (Blattberg e Deighton, 1991 - Hoffman e Novak, 1996 - Yadav e Varadarajan, 2005). Secondo alcuni contributi di ricerca, influirebbero sullo sfruttamento delle citate potenzialità:

- "l'età dell'azienda" (Kai-Uwe Brock, 2000 - MacGregor et al., 2002): le PMI presenti nel proprio settore da molto tempo e quelle di recente formazione si dimostrerebbero le più restie nei confronti di internet, per eccessivo radicamento sui tradizionali strumenti strategici le prime, e per carenza di risorse finanziarie le seconde;

- le dimensioni dell'azienda e il numero di dipendenti (Dholakia, Kshetri, 2004);

- il settore di attività prevalente (Martin e Matlay, 2001): sarebbero soprattutto le imprese operanti nel settore dei servizi a evidenziare la maggiore propensione nei confronti di internet;

- il livello di conoscenze IT diffuso all'interno dell'azienda;

- le caratteristiche ed il livello evolutivo dell'ambiente tecnologico in cui l'impresa opera;

- le percezioni e le attitudini del management;

- la strategia perseguita dall'azienda: orientamento al profitto e al breve termine influirebbero negativamente sullo sfruttamento strategico della rete;

- l'ambiente competitivo: la percezione di utilizzo di internet da parte della concorrenza e il customer service veicolato attraverso la rete, avrebbero un ruolo importante nell' adozione e declinazione delle strategie e dell'internet marketing mix (Dholakia, Kshetri, 2004).

Ancora, il contributo della copiosa letteratura ci consente di individuare i principali benefici percepiti dalle PMI in seguito all'utilizzo di internet: benefici diretti e indiretti, a breve e a lungo termine. La tabella 1 ne opera una efficace sintesi. 
Tab. 1 - Le '7C' di internet

Cost reduction: è ottenibile limitando l'utilizzo del telefono per i servizi di vendita e di marketing e sostituendo la stampa e la distribuzione del materiale di comunicazione con la sua pubblicazione sul sito web. Inoltre attraverso l'e-commerce può essere eliminato un buon numero di broker, con una riduzione dei costi di transazione (Butera, 2001).

Capability: internet fornisce nuove opportunità in quanto permette l'introduzione di nuovi prodotti e servizi, lo sfruttamento di nuovi mercati (compresi i segmenti di minori dimensioni), l'eliminazione dei vincoli geografici, la possibilità di svolgere attività di vendita e di assistenza 24 ore su $24, \ldots$. Si realizza così quanto definito da John Naisbitt (1982) come "paradosso globale": più grande diviene l'economia mondiale più forti diventano i piccoli operatori. Internet permette cioè ai piccoli imprenditori di operare su scala globale (loppolo e Scazzoso, 1999). In questo caso, tuttavia, si rende necessario un ripensamento di strategie e tattiche di marketing, sempre che queste siano state definite dalla PMI (Cedrola, 2005), oltre che una profonda riflessione sui temi della logistica e dell'ottimizzazione distributiva.

Competitive advantage: quando un'azienda introduce nuove capacità prima dei suoi concorrenti, realizzerà un vantaggio competitivo fino a che questi non raggiungeranno le stesse capacità (Stockdale e Standing, 2004).

Communications improvement: tramite internet si possono rendere più efficienti ed efficaci le comunicazioni con i clienti, con il personale, con i fornitori e con i distributori; le informazioni sono continuamente reperibili e aggiornabili velocemente e a basso costo (Kaynak e Tatoglu e Kula, 2005). Kotler parla anche di notevole riduzione delle asimmetrie informative (Kotler, 2004).

Control: Internet permette di effettuare ricerche di marketing di migliore qualità, riguardanti sia il comportamento dei consumatori che il modo di operare del personale (Prandelli e Verona, 2002).

Customer service improvement: tramite la realizzazione e la consultazione di database è possibile ottenere in tempo reale dati relativi alla disponibilità delle merci o alle richieste presentate al servizio clienti (Chaffey et al., 2001). Inoltre è ipotizzabile la collaborazione tra diverse localizzazioni di una stessa organizzazione, così come tra partner di business (Castellet, 2007). Un maggiore orientamento al cliente può poi sostanziarsi nella possibilità di personalizzare l'offerta, con particolare riferimento ai prodotti digitalizzabili, anche se questa opzione è progressivamente implementata anche dalle imprese che realizzano prodotti tangibili ${ }^{1}$.

Collaboration and information sharing: innovazione tecnologica ed evoluzione comportamentale favoriscono lo sviluppo del rapporto fra utente/consumatore e produttore/distributore, fino a tradurlo in un vero e proprio collaborazionismo. Inoltre si sviluppano approcci sociali alla generazione e distribuzione di contenuti via web caratterizzati da processi comunicativi aperti, con una forte decentralizzazione dell'autorità (ad esempio blog, community, mondi virtuali).

Le modalità mediante le quali le aziende possono creare valore attraverso il web sono numerose. In primo luogo internet favorisce il miglioramento del modello di business adottato dalle imprese, sostenendo la riduzione dei costi, il miglioramento del servizio reso al cliente in termini di ampiez-

1. Ad esempio l'offerta online di Nike prevede la possibilità di realizzare, interagendo con il sito, scarpe da ginnastica personalizzate in ogni particolare, consentendone l'acquisto in tempo reale. 


\section{E. Cedrola}

za e livello qualitativo, nonché l'attrazione di nuovi clienti. Questo è indubbiamente l'approccio più immediato e percorribile da parte di coloro che, per la prima volta, si affacciano alla rete.

Il perfezionamento dell'attuale catena del valore rappresenta un'ulteriore potenzialità offerta dall'interpretazione della propria presenza sul web, ad esempio disintermediando un canale distributivo inefficiente e sostituendolo con una soluzione maggiormente conforme alle dinamiche di mercato.

Relativamente alle strategie di marketing, la presenza in rete rende possibile anche il superamento del tradizionale trade-off tra ampiezza del mercato e livello di differenziazione informativa erogata al target di riferimento (Prandelli e Verona, 2002). Inoltre impatta in modo diretto sulle tre fasi del processo di marketing: analisi, strategia e implementazione, consentendo di superarne i vincoli tipici e di realizzare politiche totalmente focalizzate sul cliente, il quale può anche assumere un ruolo attivo sia in termini di risposta che di creazione di una relazione. La flessibilità propria di internet conferisce facoltà di colpire l'intero mercato con un sistema di offerta differenziato sul singolo cliente. Anche in un'ottica di marketing operativo, la scelta delle quattro $\mathrm{P}$ può essere personalizzata rispetto a tutto il mercato di riferimento (Cedrola, 2005).

Per quanto attiene alla fase di analisi, le nuove tecnologie permettono di raccogliere informazioni in profondità su un elevato numero di clienti attuali e potenziali che navigano e interagiscono via internet. Quanto affermato riguarda anche l'analisi dell'ambiente competitivo, facilitando la fase di avvicinamento a culture e mercati internazionali (Stockdale e Standing, 2006).

\subsection{Atteggiamento delle PMI verso la Rete e problematiche da af- frontare}

Nonostante le numerose potenzialità espresse, ad oggi le PMI hanno tendenzialmente "assistito" al processo di evoluzione della rete, lasciandosi coinvolgere solo parzialmente. In molti casi si sono invece limitate ad una mera esplorazione delle opportunità di miglioramento del business, senza precisi obiettivi, ma soprattutto senza la forza di sperimentare soluzioni veramente innovative. Questo sebbene le basse barriere di accesso in termini di costo e di conoscenze tecnologiche necessarie ponessero le piccole e medie imprese in una relazione di sostanziale parità nei confronti delle imprese maggiori. La ridotta dimensione, infatti, consente una minore complessità organizzativa che si traduce in semplificazione "strutturale" dei siti e in migliore coerenza comunicativa e di marketing. A ciò si accompagna anche una maggiore velocità decisionale. Infine, le imprese minori sono generalmente impegnate in relazioni di mercato di tipo diretto 
con la clientela, interiorizzando un approccio di comunicazione e di marketing maggiormente vicino alle potenzialità d'uso e alla cultura dell'internet marketing.

Una ricerca svolta da Vescovi (1999) su un campione di piccole e medie imprese italiane, evidenziava sei problemi ricorrenti e relativi all'introduzione di internet nel marketing mix aziendale. Gli aspetti critici individuati attengono ai seguenti temi:

- definizione di una chiara strategia di marketing;

- cambiamento delle regole e dei paradigmi di marketing;

- integrazione con gli strumenti e le attività di marketing tradizionale;

- convinzione e coinvolgimento dell'impresa nel progetto di internet marketing;

- sviluppo delle risorse umane per il marketing in internet;

- attuazione di cambiamenti nell'organizzazione di marketing.

Tali problemi rappresentano cause di difficoltà e di rallentamento all'introduzione dell'internet marketing nelle imprese minori. In molte situazioni la responsabilità delle esperienze fallimentari è in capo all'atteggiamento attendista delle aziende che si affacciano alla rete. Tale approccio si concretizza attraverso lo sviluppo di una presenza web senza chiari obiettivi né strategie, finalizzata soprattutto a testimoniare la propria esistenza. Questo atteggiamento è giustificato dal basso costo, almeno iniziale, che l'operazione comporta $\mathrm{e}$, in parte, dalla sensazione che si tratti di attività di importanza secondaria, delegabili interamente a società esterne. In tale prospettiva si rivela profondamente errata la predisposizione di "siti standard o imitativi" che, una volta allestiti, verranno abbandonati al loro destino.

Ma il ricorso ad internet costituisce anzitutto un'opportunità di marketing interattivo (Hoffman e Novak, 1996). A tal proposito Yadav e Varadajan (2005) hanno evidenziato una serie di fattori che ricevono impulso proprio dallo sviluppo dell'interazione in internet. Parliamo di: valore erogato a compratori e venditori (impatto positivo), migrazione del prodotto sul marketplace elettronico ${ }^{2}$ (impatto positivo), caratteristiche principali del prodotto (impatto positivo specialmente per prodotti digitali, deperibili e con frequenza di acquisto elevata), bisogni informativi degli acquirenti (impatto positivo), limitatezza del mercato e dispersione geografica dei consumatori (impatto positivo).

In definitiva, un'impresa che possa veramente dirsi "internet oriented" deve concepire la presenza sul web come una componente della struttura d'azienda, quindi integrarla con tutti i settori aziendali. Da ciò derivano le difficoltà legate alla reingegnerizzazione del proprio business e alla neces-

2. Componenti della migrazione di prodotto: migrazione informativa, migrazione delle transazioni e degli ordini, migrazione delle attività di supporto e post-acquisto. 


\section{E. Cedrola}

sità di riqualificare il personale affinché sia preparato ad affrontare la nuova realtà. Tali processi sono più semplici per le piccole aziende, perché esse incontrano meno difficoltà ad integrare internet nei propri sistemi organizzativi, di promozione e vendita (Veragouth, 2000).

Inoltre è necessario che l'azienda abbia chiari i propri obiettivi, il proprio target e le proprie strategie (Amor, 2000). È infatti un grosso errore fare ingresso in rete perché tutti sono presenti, oppure perché ciò può conferire all'azienda un'immagine di modernità. Questo approccio molto comune è di tipo reattivo e non proattivo (Chaffey et al., 2001) e implica un erroneo ribaltamento delle priorità: partire dalla soluzione invece che dal problema, dagli strumenti invece che dagli obiettivi. Si considerano cioè le tecnologie e come potrebbero essere usate e non invece lo specifico problema e il modo migliore di risolverlo.

Per dare ulteriore concretezza alle osservazioni finora proposte, nei paragrafi che seguono verranno esposti e brevemente commentati i risultati di un watching su un campione di siti di PMI. Lo scopo che detta analisi si propone è quello di verificare le tipologie di utilizzo di internet da parte delle PMI italiane, operando successivamente un confronto a livello europeo. Inoltre si prefigge, come secondo obiettivo, di individuare le aree di miglioramento e di potenziale sviluppo.

La metodologia utilizzata per la realizzazione di tale indagine, che verrà di seguito dettagliata, prende spunto da un modello di valutazione elaborato e testato per l'analisi di altri ambiti imprenditoriali quali i settori finanziario, ferroviario, postale e industriale (Cedrola, 2002 (a)-2002 (b)-2003-2004).

Per quanto attiene alla struttura, il lavoro focalizza dapprima l'attenzione sullo stato dell'arte delle PMI italiane in rete, alla luce delle evidenze empiriche del watching 2004. Viene poi presentata l'evoluzione riscontrata a distanza di due anni, concentrando l'attenzione su quello che è stato definito l' "orientamento al cliente dei siti internet". Tale disamina è stata realizzata con l'ausilio di un sistema di ponderazione volto a valorizzare e a rendere possibile la comparazione tra gli attributi rilevati. Successivamente è analizzato il ricorso alla rete da parte di un campione di PMI europee (rilevazione 2007) al fine di individuare similitudini e differenze di approccio. Vengono poi evidenziate le aree di miglioramento principali e le aree di sviluppo auspicabili.

\section{La presenza in Rete delle PMI italiane: lo stato dell'arte}

L'obiettivo principale dell'indagine è consistito nel comprendere il ruolo attribuito ad internet da parte delle PMI italiane. A questo scopo è stato analizzato un campione di 270 siti di imprese piccole e medie suddivise in 10 settori di attività: abbigliamento, agricoltura caccia e pesca, alimentari e 
bevande, alberghi e ristoranti, costruzioni, materiali per l'edilizia, elettronica, meccanica, trasporti e servizi ${ }^{3}$. Si tratta in tutti i casi di società di capitale con numero di dipendenti inferiore a 500, la cui attività prevalente è espressa nelle seguenti proporzioni: $57 \%$ produzione, $19 \%$ vendita all'ingrosso e $24 \%$ vendita al dettaglio. Le osservazioni sono state svolte nel periodo giugno-agosto 2004 e ripetute nei mesi di giugno-agosto 2006. In questo secondo watching è stata possibile la visita di soli 237 siti. Dopo un biennio ben 33 web sites sono risultati irrintracciabili in rete oppure in aggiornamento. Nonostante ciò, la numerosità percentuale per settore e regione è rimasta pressoché immutata, consentendo la comparazione dei risultati ottenuti nelle due annualità di rilevazione.

Ai fini poi di verificare similitudini o distanze di approccio tra imprese italiane ed europee, nel periodo giugno-settembre 2007 è stato svolto un terzo watching su un campione di 549 imprese (tab. 2). Per quanto riguarda l'Italia sono stati utilizzati siti già analizzati nelle precedenti ricerche.

Tab. 2 - II campione di imprese europee

\begin{tabular}{lrlr}
\hline Settore di appartenenza & $\%$ & Paese & Numerosità \\
\hline Agricoltura caccia e pesca & $7 \%$ & Regno Unito & 146 \\
Alberghi e ristoranti & $7 \%$ & Germania & 125 \\
Alimentari e bevande & $7 \%$ & Francia & 98 \\
Calzature e pelli & $1 \%$ & Spagna & 90 \\
Costruzioni e materiali per l'edilizia & $11 \%$ & Italia & 90 \\
Editoria carta e prodotti in carta & $1 \%$ & & \\
Elettrodomestici ed elettronica & $10 \%$ & & \\
Farmaceutica Chimica e vetro & $2 \%$ & & \\
Meccanica & $11 \%$ & & \\
Mobili legno e prodotti in legno & $2 \%$ & & \\
Servizi & $10 \%$ & & \\
Tessile e abbigliamento & $8 \%$ & & \\
Trasporti & $4 \%$ & & \\
Viaggi e turismo & $12 \%$ & & \\
Altro & $6 \%$ & & \\
\hline
\end{tabular}

Per la valutazione dei siti è stato realizzato un questionario a risposte chiuse $^{4}$ suddiviso in sette sezioni principali: le leve del marketing mix

3. Per l'individuazione del campione rappresentativo sono stati analizzati i dati di Movimprese delle società di capitale registrate nel quarto trimestre 2003, operanti in 45 macro settori. Il campione estratto è rappresentativo per macrosettori e per regione italiana. Sono state selezionate soltanto società di capitali perché è stato effettuato un primo tentativo di esaminare la correlazione esistente tra valutazione del sito internet e solidità aziendale. I dati di bilancio per effettuare l'analisi sono stati ricavati dalla banca dati di Lince S.p.A.

4. Nella maggior parte dei casi si tratta di risposte dicotomiche; solo in alcuni casi sono state utilizzate scale di valutazione a più alternative di scelta. Il questionario evidenzia un livello di approfondimento elevato del tema indagato, in quanto consta di circa 100 domande. 


\section{E. Cedrola}

(prodotto, comunicazione istituzionale, prezzo, distribuzione), la relazione e l'interazione con la clientela ed i visitatori, le caratteristiche strutturali e stilistiche dei siti e la funzione informativa. La tabella 3 riassume le principali tematiche oggetto di analisi.

Nei paragrafi che seguono verranno esposti e brevemente commentati i principali risultati relativi alle aree tematiche individuate (dati 2004). Saranno poi evidenziati gli sviluppi ed i principali scostamenti derivanti da modifiche dei siti intervenute a distanza di due anni (dati 2006). In chiusura del lavoro verrà proposta una valutazione globale circa la corretta interpretazione della presenza in rete da parte delle PMI italiane, in ottica di orientamento al mercato ed alla relazione, e verrà operato un confronto con la situazione di un gruppo di imprese spagnole, francesi, tedesche ed inglesi analizzate (dati 2007).

Tab. 3 - Schema tematico della ricerca

\begin{tabular}{|c|c|}
\hline $\begin{array}{l}\text { Presentazione } \\
\text { dell'azienda e } \\
\text { caratteristiche } \\
\text { del sito Internet }\end{array}$ & $\begin{array}{l}\text { - Disponibilità di informazioni sull'impresa ed } \\
\text { altre informazioni } \\
\text { - Caratteristiche strutturali e stilistiche dei siti } \\
\text { - Navigabilità dei siti }\end{array}$ \\
\hline Prodotto & $\begin{array}{l}\text { - Segmentazione e disponibilità informativa sui } \\
\text { prodotti } \\
\text { - Personalizzabilità dei prodotti }\end{array}$ \\
\hline Comunicazione & $\begin{array}{l}\text { - Utilizzo di pubblicità on-line } \\
\text { - Utilizzo di promozioni on-line } \\
\text { - Partnership con altre organizzazioni }\end{array}$ \\
\hline Pricing & $\begin{array}{l}\text { - Disponibilità di informazioni sui prezzi } \\
\text { - Personalizzabilità dei prezzi sul cliente } \\
\text { - Tipologie di pagamento }\end{array}$ \\
\hline Canale di vendita & $\begin{array}{l}\text { - Disponibilità di informazioni sui punti vendita } \\
\text { - Possibilità di effettuare ordini on-line } \\
\text { - Possibilità di effettuare pagamenti on-line } \\
\text { - Distribuzione dei prodotti on-line }\end{array}$ \\
\hline Relazione con la clientela & $\begin{array}{l}\text { - Disponibilità di servizi personalizzati } \\
\text { - Disponibilità di un servizio clienti } \\
\text { - Comunicazioni on-line alla clientela } \\
\text { - Creazione di comunità, di forum di discussione } \\
\text { e blog }\end{array}$ \\
\hline
\end{tabular}




\subsection{L'informativa sull'azienda e la struttura del sito}

La prima sezione della ricerca riguarda sia la presentazione dell'impre$s a$, sia l'aspetto e la struttura del sito internet, allo scopo di comprendere quanto, in che modo e con quale grado di approfondimento, le piccole e medie imprese utilizzino la rete per diffondere informazioni sull'azienda e sulla propria attività. Dall' analisi dei risultati ottenuti nell'osservazione del 2004 si può notare che l' $84,1 \%$ delle aziende prevede una sezione dedicata alla storia o presentazione dell'azienda, anche se con un approccio molto sintetico. Vengono infatti diffuse informazioni sulla storia, missione e valori d'impresa nel $28,5 \%$ dei casi analizzati, sulle iniziative promosse dall'azienda nel $24,1 \%$, informazioni sull'andamento economico dell'azienda nel $58,5 \%$.

In riferimento a quest'ultimo, le notizie più diffuse sono relative alle principali linee produttive $(48,5 \%)$, ai mercati di riferimento $(26,3 \%)$ e alla certificazione qualità $(25,9 \%)$. Tali percentuali medie non rispecchiano il comportamento di tutti i settori di attività analizzati (dettaglio esposto in tabella 4). Stupisce la carenza informativa sui dati di fatturato e di bilancio, che interessa uniformemente sia le imprese con migliori indicatori di performance aziendale, sia quelle con difficoltà di mercato. Questo è un chiaro segnale di maggiore attenzione rivolta al consumatore finale. In questo caso è infatti importante far percepire al navigatore un'immagine di affidabilità: la presenza di una sezione dedicata alla storia dell'azienda, anche se minimale, nonché una struttura del sito che tiene conto delle principali regole del web marketing, sono in grado di supportarla, rassicurando il navigatore ed evitando di fornire informazioni che potrebbero risultare ridondanti o fuorvianti.

Notizie aggiuntive sull'impresa sarebbero invece auspicabili in ambito $B t o B$, in particolare per quelle imprese già presenti o con potenziale interesse per i mercati internazionali. In effetti, in questo campo già alcuni passi sono stati compiuti: è stata segnalata la possibilità di consultare il sito in altre lingue nel $42,6 \%$ dei siti esaminati. Anche in questo caso si sono rilevate differenze importanti tra settori, con valori molto elevati per alberghi e ristoranti $(91,7 \%)$, materiali per l'edilizia $(87,5 \%)$ e abbigliamento $(75 \%)$, e valori molto bassi per trasporti $(12,5 \%)$ e costruzioni $(21,4 \%)$.

Nel questionario utilizzato per il monitoraggio dei siti sono stati dedicati alcuni quesiti alla struttura, alla tipologia di grafica ed alle immagini utilizzate, la cui scelta si rileva di particolare importanza per conferire concretezza e tangibilità non solo al servizio, ma anche al rapporto a distanza. Ben il 91,5\% delle imprese fa ricorso ad immagini fisse e a fotografie, mentre meno utilizzate sono le icone $(48,9 \%)$ e i testi scorrevoli $(28,5 \%)$, nonostante questi ultimi rivelino una notevole efficacia per richiamare e focalizzare l'attenzione su quelle notizie che non devono rimanere inosserva- 


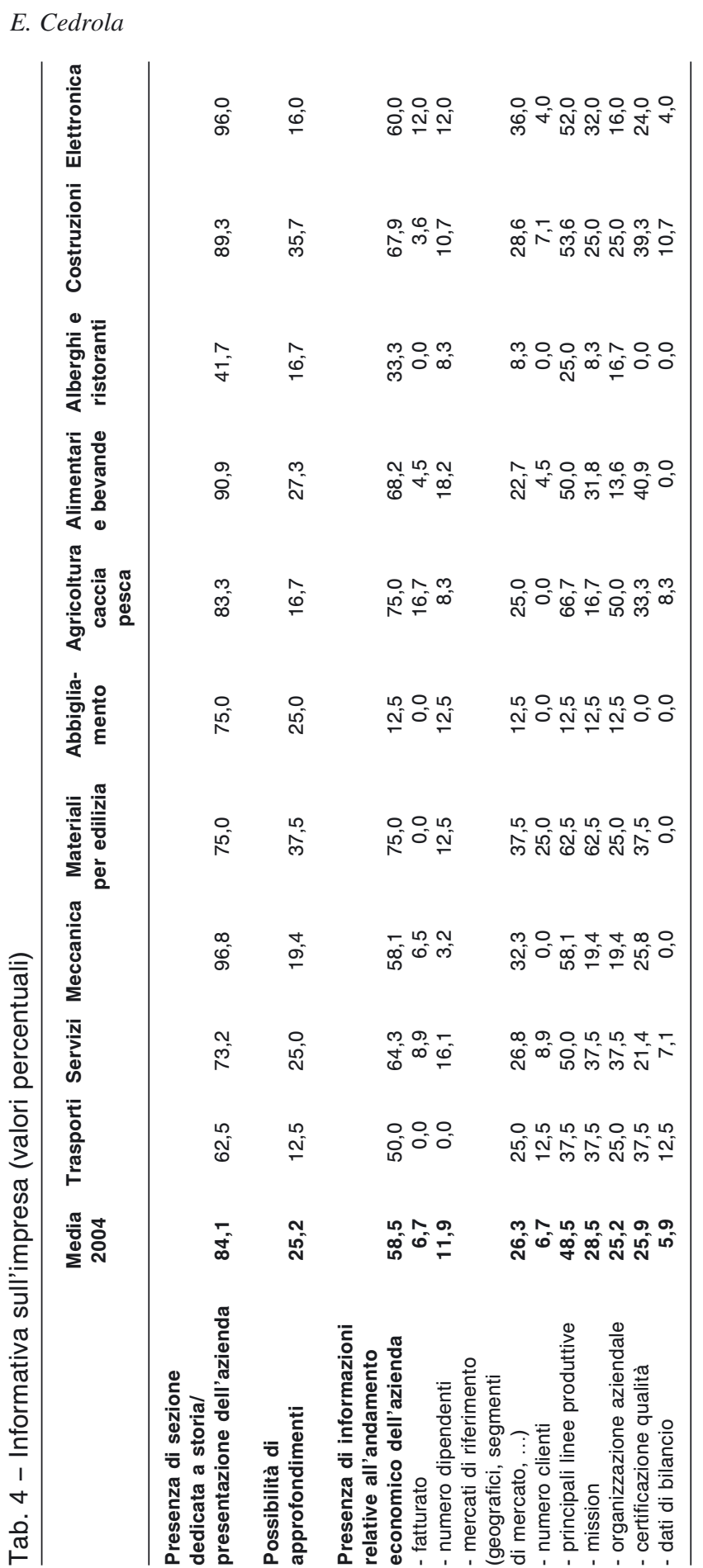


te. Situazioni di maggiore ricorso ad immagini e fotografie vengono espresse dai settori dell'abbigliamento, agricoltura caccia e pesca.

Passando a considerare gli elementi strettamente legati alla comunicazione via internet emerge una situazione piuttosto buona: si è riscontrata la presenza di barra di navigazione nel 52,6\% dei siti, di menù $(81,5 \%)$, di link ben riconoscibili che consentono il ritorno alla home page $(75,2 \%)$. Mappe e motori di ricerca sono invece meno impiegati, con percentuali di presenza variabili tra il $20,7 \%$ e il $13,3 \%$. In questo caso il comportamento è simile in tutti i settori esaminati. Tendenzialmente nei siti viene utilizzata una grafica omogenea $(89,6 \%)$, i titoli e le parti importanti dei testi sono evidenziate in grassetto, con dimensioni e colori differenti $(92,6 \%)$.

In definitiva è possibile rilevare una situazione decisamente buona per quanto concerne la struttura dei siti, mentre maggiore attenzione dovrà essere dedicata alla funzione informativa, vista l'abitudine ormai consolidata di ricercare informazioni di ogni tipo in internet.

\subsection{La declinazione del marketing mix online}

La ricerca si è posta anche l'obiettivo di rilevare quanto e come i siti web delle imprese campione utilizzino la rete per far conoscere i propri prodotti e, soprattutto, come sfruttino le potenzialità del mezzo per segmentare il mercato, personalizzare la propria offerta, distribuire i prodotti e instaurare una relazione con i clienti attuali e potenziali. I dati raccolti hanno messo in luce come il $51,1 \%$ dei siti analizzati abbia intrapreso la via della segmentazione del mercato online seguendo diversi approcci, coerenti con il tipo di prodotto e di business. La tipologia di prodotto fornito è la modalità di segmentazione più utilizzata, evidenziando l'approccio strategico prevalente di queste imprese: l'orientamento al prodotto ovvero alle linee di prodotto, piuttosto che ai clienti.

Per quanto attiene alla disponibilità informativa sulla gamma di prodot$t i$ offerti, lo scenario generale è molto buono: in media 83 siti su 100 dettagliano queste informazioni, con situazioni di eccellenza relative ai settori dei materiali per edilizia, alberghi e ristoranti (100\%). Purtroppo i risultati sono meno incoraggianti circa la personalizzabilità dei prodotti su gruppi di consumatori/segmenti di mercato $(23 \%)$ e su singoli individui $(21,1 \%)$, ottenuti selezionando o richiedendo specifiche opzioni di prodotto. Un'eccezione è stata riscontrata nel settore dei trasporti in cui tale possibilità è contemplata nel $50 \%$ dei siti analizzati.

Se ne deduce la necessità di un maggiore sfruttamento delle potenzialità di personalizzazione delle offerte. La difficoltà, come precedentemente espresso, è quella di adeguare la struttura organizzativa aziendale, la quale deve essere in grado di recepire ed implementare tempestivamente le istan- 


\section{E. Cedrola}

ze provenienti dal mercato e veicolate dalla rete. Questo atteggiamento rilevato in rete è il riflesso di una strategia aziendale orientata più al prodotto che al cliente ed alla relazione, o addirittura dell'assenza di disegno strategico.

Sfruttare le potenzialità espressive di internet significa saper promuovere adeguatamente, anche attraverso la comunicazione pubblicitaria onli$n e$, il proprio marchio, i propri prodotti e il proprio sito (Zeff e Aronson, 1999). Nella sezione del questionario dedicata alla comunicazione ci siamo prefissi di studiare i siti che realizzano comunicazioni pubblicitarie online e le modalità con le quali vengono implementate (presenza o meno di banner, pop up, prodotti in evidenza, promozioni on e off line, attività di co-marketing). I dati raccolti indicano che ci troviamo in presenza della leva meno sfruttata nell'ambito dell'internet marketing mix. Solo 1'8,9\% del campione fa pubblicità online dei propri prodotti. Una percentuale più alta di utilizzo viene espressa dai settori abbigliamento, agricoltura caccia e pesca $(25 \%)$. Praticamente inesistente è la possibilità di personalizzare le comunicazioni sulle caratteristiche del singolo navigatore $(1,1 \%)$, segno questo di un approccio ancora statico e tradizionale allo strumento.

In tema di promozione, i siti sono indubbiamente i nuovi veicoli promozionali per l'azienda, potendo implementare online tutte le strategie promozionali già utilizzate nel mondo fisico: promozioni reduced loss ed extra gain, sia a vantaggio immediato che differito. Per quanto concerne i siti analizzati, si rileva come solo il $12,6 \%$ del campione realizzi campagne promozionali in rete. Rappresentano un'eccezione i settori alimentari e bevande e alberghi e ristoranti, che fanno registrare percentuali pari a $31,8 \%$ e $41,7 \%$ rispettivamente. La quasi totalità delle promozioni adottate riguardano sconti diretti sui prodotti dell'azienda $(13,4 \%)$, mentre scarsamente utilizzati sono sconti su prodotti di altre imprese, vendite agevolate su grossi quantitativi e prodotto/servizio del mese. Sicuramente quest'area è suscettibile di importanti modifiche, in virtù delle maggiori possibilità di personalizzazione e innovazione rese possibili dalle nuove tecnologie.

Il sito web può configurarsi, oltre che come piattaforma comunicativa, anche come nuovo canale di vendita alternativo o sinergico alla tradizionale rete distributiva. Da un attento esame dei siti è possibile osservare uno scarso ricorso a tale funzione. La percentuale media è del $8,9 \%$, mentre i settori maggiormente impegnati in tale ambito sono quello dei trasporti $(25 \%)$ e degli alberghi e ristoranti (33,3\%). Molto superiore è l'utilizzo medio per comunicare informazioni sui singoli punti vendita $(33,8 \%)$ e per erogare servizi online $(38,5 \%)$. La tabella che segue (tab. 5) mette in evidenza i risultati dell'indagine, a sostegno della convinzione che il sito web raccolga ancora, tra le PMI italiane, manifestazioni di scarso interesse, sia 


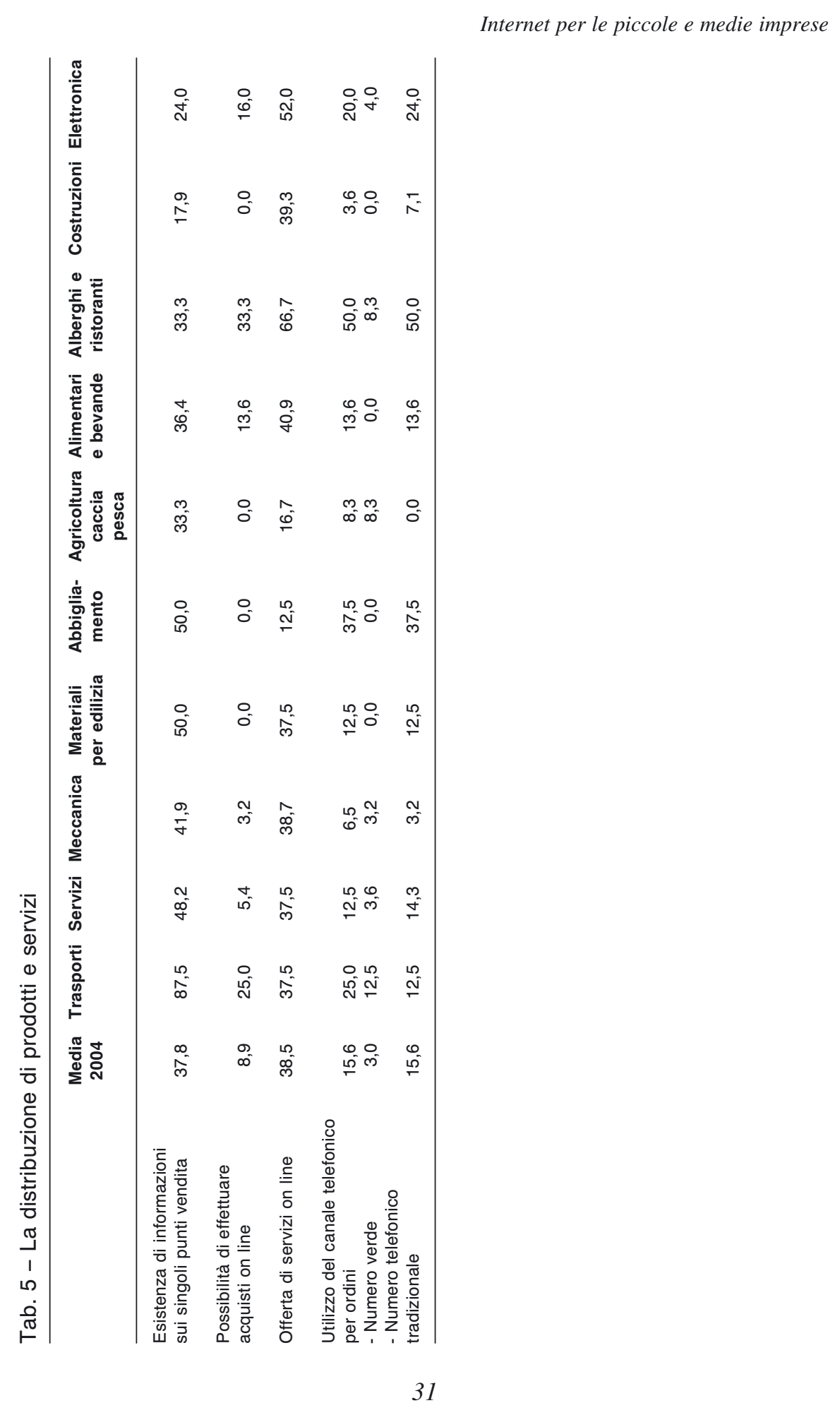




\section{E. Cedrola}

nei confronti degli impieghi più tradizionali (quali la distribuzione) che delle attività maggiormente innovative ${ }^{5}$.

Per quanto attiene alla segnalazione dei punti di vendita e ai servizi online, si riconfermano settori di spicco i trasporti e gli alberghi e ristoranti, che più di altri hanno recepito le opportunità del mercato e le istanze di alcune tipologie di clientela, particolarmente propense alla ricerca di informazioni e all'acquisto su internet.

Infine, in merito al pricing, abbiamo constatato che solo il $23 \%$ del campione fornisce informazioni chiare sui prezzi di prodotti e servizi. Questo risultato medio accomuna quasi tutti i settori, ad eccezione di quello degli alberghi e ristoranti in cui la percentuale sale all' $83,3 \%$. La differenza può essere spiegata dalla tipologia di offerta (il cliente di queste imprese ormai attende la disponibilità diffusa di queste informazioni), nonché dalle prassi operative e gestionali delle PMI italiane (Cedrola, 2005 - Namiki, 1988). Il riferimento è fatto, in particolare, alla flessibilità produttiva fondata sulle specifiche di prodotto individuate da ogni cliente, che rende maggiormente impegnativa e "compromettente" la definizione dei prezzi da pubblicare in rete. I comportamenti invece sono uniformemente condivisi nel caso di personalizzazione dei prezzi sul singolo cliente, presente solo nel 6,7\% dei casi, o di possibilità di negoziare i prezzi online (1,5\%).

Anche per quest'ultima leva i miglioramenti possibili sono numerosi, come si evince dalle evidenze empiriche e dagli orientamenti aziendali diffusi.

\subsection{Internet quale strumento relazionale}

L'ultima sezione dell'indagine affronta la delicata questione delle "relazioni con la clientela". Dalle indicazioni emerse è chiaro che le PMI italiane presenti in rete avranno, nell'immediato futuro, molta strada da percorrere sulla via dell'orientamento al cliente. In particolare gli sforzi maggiori dovranno essere rivolti a conoscere e ad inserire all'interno dell'internet marketing mix gli strumenti più innovativi, tipici della comunicazione digitale. Per quanto concerne invece gli ambiti più diffusi, dovrà essere dato spazio all'improvement. Ad esempio, la possibilità di ottenere dal sito l'erogazione di servizi personalizzati è contemplata nel $41,9 \%$ dei casi analizzati (picchi di frequenza sono stati evidenziati nei settori trasporti $(62,5 \%)$ ed elettronica $(64 \%)$ ). La disponibilità di un servizio clienti online è maggiormente diffusa (54 siti su 100) e viene realizzata prevalentemente mediante posta elettronica $(51,5)$. Trascurate sono invece le altre modalità di

5. Per quanto concerne i servizi online si tratta di proposte molto tradizionali: si sostanziano nella disponibilità di newsletters o di omaggi relativi ai prodotti commercializzati. 
interazione come numeri verdi e telefono $(5,9 \%)$ o prenotazione di colloqui con il personale dell'azienda $(3,7 \%)$.

Tra gli strumenti maggiormente pervasivi e con più alti contenuti di relazione dovranno essere attentamente valutati e realizzati: aree di discussione online $(8,1 \%)$, forum di discussione $(2,2 \%)$ e sezioni dedicate alla raccolta e risoluzione delle problematiche relative all'utilizzo dei prodotti offerti $(3,7 \%)$. Da valutare potrebbero essere anche i blog, tuttavia la scarsità di risorse finanziarie disponibili, unita alla necessità di competenze e risorse umane dedicate, impongono un sostanziale ridimensionamento del loro ruolo. In effetti, nel watching realizzato non sono stati riscontrati esempi applicativi. Diversa è la situazione delle imprese di maggiori dimensioni, in cui il rilievo dato al cliente e alla relazione con vari pubblici ha indotto numerose aziende a prevedere strumenti innovativi ed interattivi ${ }^{6}$.

Nel paragrafo che segue verrà presentato l'orientamento al cliente dei siti internet esaminati. Successivamente sarà operato un confronto con le evidenze del watching effettuato a distanza di due anni, al fine di verificare non solo l'evoluzione, ma anche le direzioni di miglioramento che sono state intraprese dalle PMI italiane.

\section{L'orientamento al cliente delle PMI italiane su internet: evoluzioni nel biennio 2004/2006}

A questo punto dell' analisi, è possibile tradurre le valenze insite in ogni attributo analizzato in "vettori" di orientamento al cliente, nonché fare il punto della situazione sul livello evolutivo raggiunto dalle PMI esaminate nello sfruttamento delle peculiarità di internet. $\mathrm{Al}$ fine di concretizzare tale intendimento è stato definito un sistema di ponderazione dei dati che consentisse, in prima istanza, di riunire in categorie omogenee le varie tematiche osservate e, in secondo luogo, di apprezzare i singoli ambiti operativi con scale di valutazione omogenee e comparabili. Tale operazione rende altresì possibili valutazioni differenziali tra situazione attuale e situazione auspicabile, in un'ottica di maggior soddisfazione delle esigenze della clientela. Il citato sistema di ponderazione, definito alla luce dei principali contributi della letteratura sull'internet marketing, individua anzitutto sette macro gruppi di variabili:

- la funzione informativa, che riguarda sia notizie sull'azienda sia informazioni di vario genere ed utilità;

- la struttura, sezione che attribuisce un giudizio circa la capacità di rendere agevole, ma nel contempo attraente, la navigazione del sito;

6. Un ottimo esempio è rappresentato dal blog inserito nel sito di Patagonia www.patagonia.com (ultimo accesso 18/11/2008). 


\section{E. Cedrola}

- il prodotto, la comunicazione, il prezzo, la distribuzione, che contengono una valutazione sull'adattamento del modello di business all'operatività virtuale e multi-canale;

- la relazione, che focalizza l'attenzione sull'utilizzo del web per la costruzione di rapporti one to one con la propria clientela e con i navigatori in genere.

A ciascuna variabile classificata nelle singole macro-aree è stato successivamente attribuito un peso, che ha consentito l'elaborazione del grafico riepilogativo proposto in figura 1 , che pone a confronto la situazione del 2004 con quella del watching 2006.

Fig. 1 - Stato dell'arte delle PMI italiane in Rete

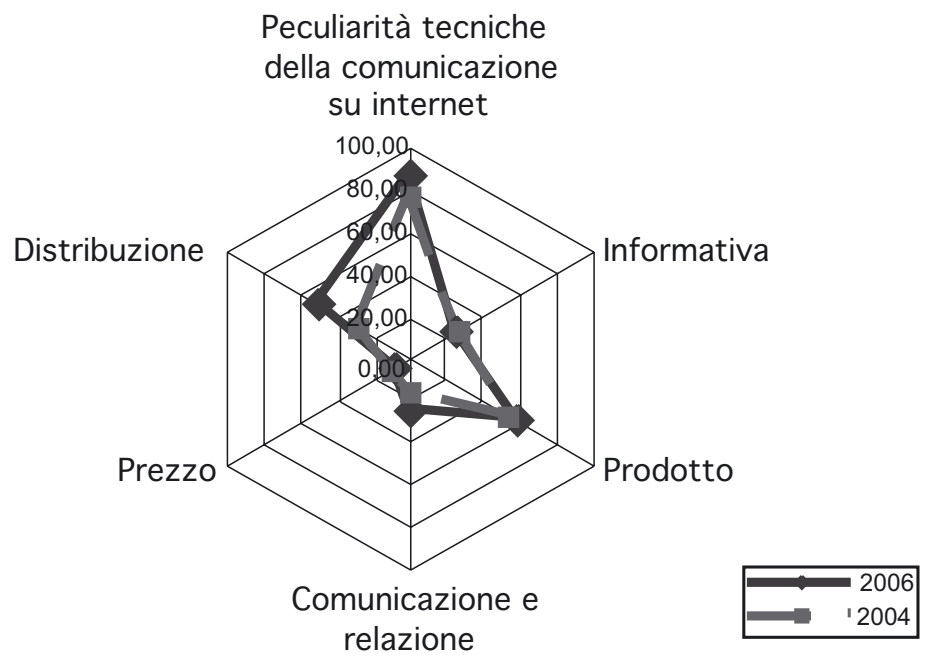

Se ne ottiene, anzitutto, un'indicazione di elevata attenzione posta nei confronti delle peculiarità tecniche della comunicazione su internet. In effetti tutti i settori analizzati con la medesima metodologia (finanziario, ferroviario, postale) hanno evidenziato i primi risultati positivi in questo ambito (Cedrola 2002a, 2002b, 2003, 2004), mostrando un ricorso corretto alla rete, nel senso che vengono rispettate le principali regole per la definizione di un buon sito internet. La motivazione va ricercata soprattutto nelle scelte organizzative legate al tema web. Nella maggior parte dei casi infatti, i siti vengono affidati per sviluppo e manutenzione a società esterne, che se da un lato assicurano la correttezza formale, dall'altro non sono in grado di garantire la vitalità dello strumento, anche in considerazione delle limitate risorse destinate a internet da parte delle imprese. 
La funzione informativa presenta ancora numerosi limiti, tuttavia, rispetto alla situazione 2004, nel 2006 si sono registrati lievi sviluppi, sia in tema di pubblicazione di informazioni sull'andamento economico dell'azienda, sia di evidenza conferita alle iniziative promosse dall'azienda. Migliorata è anche la traduzione dei siti in altre lingue, soprattutto l'inglese $(48,10 \%)$. Questo trend non può che influire positivamente sull'orientamento ai mercati internazionali delle nostre imprese. In questo senso gioca un importante ruolo anche lo spazio accordato alla variabile prodotto. In effetti si sono evidenziati progressi sostanziali sia per quanto concerne la segmentazione del sito, che in merito alla personalizzabilità dei prodotti su gruppi di consumatori e sui singoli individui. I contesti decisamente più arretrati, che hanno mantenuto la propria posizione nei due anni di osservazione, sono quelli della comunicazione e del pricing, per i quali non sono stati riscontrati cambiamenti. Se ne deduce un'assenza di variazioni sostanziali a livello strategico, sia in senso stretto (internet in quanto tale) che in senso lato (definizione e perseguimento di una precisa strategia aziendale e realizzazione di piani di marketing fondati e formalizzati).

Si nota invece un'evoluzione positiva in ambito distributivo, dove si riscontra un tentativo di integrazione con i canali tradizionali. Sono incrementate del 50\% le informazioni sui singoli punti di vendita, del 30\% l'offerta di servizi online e del $15 \%$ la possibilità di ottenere dal sito servizi personalizzati. Probabilmente questo è un segnale di inversione di tendenza: sempre più le imprese sono coinvolte direttamente nell'aggiornamento del sito e nell'erogazione diretta di servizi. In effetti la disponibilità di un servizio clienti per chi visita il sito è accresciuto dal $53 \%$ al $98 \%$, implicando nel $92 \%$ dei casi la disponibilità di un indirizzo di posta elettronica con il quale interloquire. Le analisi mettono anche in luce il diverso livello di sviluppo dell'internet marketing nei vari settori di appartenenza dei siti campione (fig. 2).

I campi di attività più tradizionalmente sviluppati, sia sotto il profilo della struttura che della distribuzione veicolata dal web sono e permangono quello dei trasporti e alberghi e ristoranti, anche se altri settori (ad esempio materiali per l'edilizia) manifestano uno stadio evolutivo "a macchia di leopardo". Possiamo dunque trarne che le opportunità nel campo dell'innovazione di prodotto, di processo e di mercato citati nel paragrafo introduttivo sono ancora ampiamente inesplorate. La funzione informativa nei confronti della clientela finale è poco sviluppata e le situazioni di commercio elettronico molto ridotte. Il ruolo di internet all'interno delle strategie di marketing è ancora minimale, anche a ragione del fatto che le PMI italiane gestiscono i canali, gli strumenti e le politiche di marketing in maniera passiva, senza un vero processo di pianificazione strategica strutturato (Cedrola, 2005). 


\section{E. Cedrola}

Fig. 2 - L'orientamento al cliente delle PMI italiane in rete

(settori/2004)

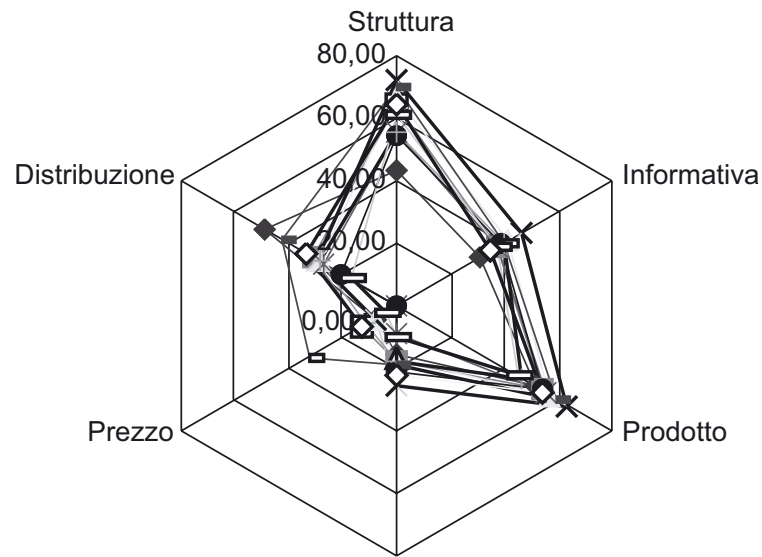

Comunicazione e relazione

\begin{tabular}{|c|c|}
\hline $\begin{array}{l}\longrightarrow \text { Trasporti } \\
\longrightarrow \square \text { Meccanica } \\
\longrightarrow \text { Abbigliamento } \\
\longrightarrow \text { Alimentari e bevande } \\
\longrightarrow \text { - Costruzioni }\end{array}$ & $\begin{array}{l}\longrightarrow \square-\text { Servizi } \\
\longrightarrow * \text { Materiali per edilizia } \\
\longrightarrow-\text { Agricoltura caccia pesca } \\
\longrightarrow-\text { Alberghi e ristoranti } \\
\sim-\text { Elettronica }\end{array}$ \\
\hline
\end{tabular}

(settori/2006)

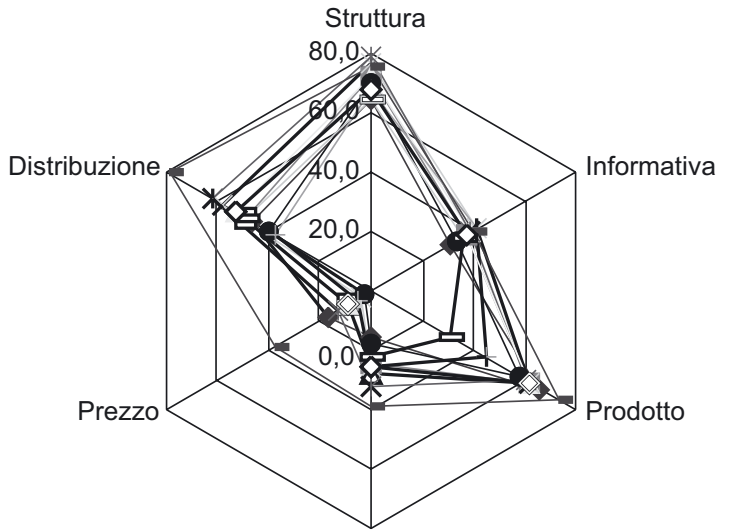

Comunicazione e relazione

$\begin{array}{lll}\longrightarrow \text { Trasporti } & -\square-\text { Servizi } & -\square \text { Meccanica } \\ \longrightarrow \text { - Materiali per l'edilizia } & \longrightarrow \text { Abbigliamento } & - \text { Agricoltura, caccia, pesca } \\ \longrightarrow \text { Alimentari e bevande } & \longrightarrow \text { - Costruzioni } & - \text { Alberghi e ristoranti } \\ \longrightarrow \text { Elettronica } & & \end{array}$


Al di là dei valori medi totali e di settore, è stata operata una ponderazione per ogni sito analizzato, ottenendo la dispersione rappresentata nella figura 3. Per la sua realizzazione, le principali tematiche analizzate dal questionario sono state suddivise in due aree di analisi:

- struttura ed informativa, a cui vengono ricondotti gli aspetti relativi alle più corrette tecniche di comunicazione in rete, nonché alla funzione di erogazione di informazioni;

- internet marketing mix, che implica una valutazione del sito internet in chiave di marketing, esplorandone la capacità di veicolare le variabili prezzo, comunicazione, distribuzione, prodotto e relazione/interattività.

Fig. 3 - I siti internet delle PMI italiane tra forma e marketing

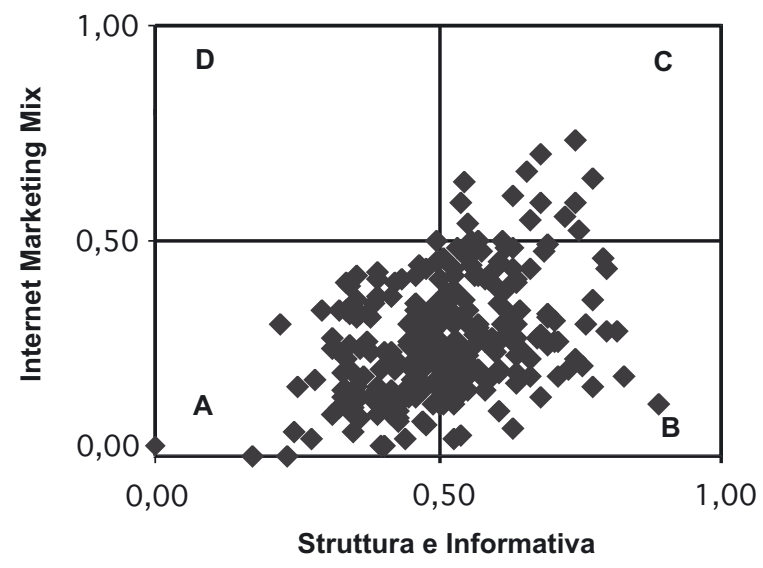

Ciascun punto rappresentato nella dispersione esprime la valutazione relativa ad ogni azienda del campione, rispetto all'impostazione del sito e alla funzione informativa assolta (ascisse) ed alla declinazione dell'internet marketing mix (ordinate). Il grafico evidenzia con chiarezza i principali ambiti di attenzione delle PMI italiane: in primis la struttura e la funzione informativa, poi l'inserimento di internet in una logica di marketing e di relazione con il cliente. È dunque ipotizzabile il seguente percorso evolutivo

$$
\mathrm{A} \rightarrow \mathrm{B} \rightarrow \mathrm{C}
$$

in cui le aziende consolidano prima la prassi e poi la strategia, anche se questo terzo passaggio verrà realizzato solo da quelle imprese che avranno compreso l'importanza dell'analisi dei mercati e della pianificazione. Quest'affermazione non è vincolata al settore di appartenenza o alla dimensione d'impresa, bensì alla convinzione e alla volontà del management di trasformare l'azienda in soggetto proattivo e non solo reattivo. 


\section{E. Cedrola}

\section{PMI italiane ed europee a confronto: similitudini e differenze}

Dal punto di vista dell'innovazione e delle applicazioni di marketing, spesso si guarda oltre oceano, o quantomeno oltralpe, per scoprire e studiare nuovi modi per proporsi ai mercati. Questo è avvenuto anche per la presenza in rete: gli Stati Uniti sono stati espressione dell'introduzione di internet nel business delle imprese, come pure di nuove soluzioni. Anche il Nord Europa si è spesso fatto portavoce di innovazioni di marketing, tanto da stimolare l'attenzione della ricerca ad esplorare l'utilizzo della rete da parte delle PMI del Regno Unito e non solo.

Proprio con l'intendimento di dare un contributo al dibattito aperto in letteratura, è stato costruito ed analizzato un campione di 549 piccole e medie imprese europee così composto: Regno Unito 145, Germania 124, Francia 97, Spagna 89, Italia 89. Le imprese sono state individuate rispettando la stessa rappresentatività di settore definita per le analisi delle imprese italiane svolte nel 2004 e 2006, ed è stato utilizzato per il watching lo stesso questionario e la stessa metodologia di valutazione.

L'analisi svolta sui valori medi evidenziava, in prima istanza, una forte similitudine comportamentale tra piccole e medie imprese (tab. 6), come pure una lievemente migliore "interpretazione" dell'internet marketing da parte delle imprese del Regno Unito, soprattutto per quanto concerne la leva distributiva.

Tab. 6 - Una valutazione sintetica/100 delle PMl europee in rete

\begin{tabular}{lcccccc}
\hline & $\begin{array}{c}\text { Struttura } \\
\text { sito }\end{array}$ & Informativa & Prodotto & $\begin{array}{c}\text { Comunicazione } \\
\text { e Relazione }\end{array}$ & Prezzo & Distribuzione \\
\hline Francia & 70 & 45 & 61 & 17 & 17 & 34 \\
Germania & 71 & 44 & 59 & 19 & 21 & 40 \\
Italia & 69 & 42 & 57 & 23 & 18 & 39 \\
Regno Unito & 71 & 41 & 62 & 23 & 28 & 45 \\
Spagna & 64 & 41 & 50 & 15 & 12 & 29 \\
\hline
\end{tabular}

Un ulteriore punto comune veniva riscontrato, a conferma della letteratura citata (Martin e Matley, 2001), nel migliore sfruttamento di internet da parte delle imprese di servizi, in particolare alberghi e ristoranti e le aziende operanti nel settore turistico.

Risultava tuttavia fondamentale concentrare l'attenzione, più che sui valori medi, sull'operato delle singole aziende. È stata dunque riproposta, anche per le 549 imprese europee, la medesima ponderazione utilizzata per giungere all'elaborazione relativa al mercato italiano (fig. 3). La situazione delle imprese europee è esposta nella figura 4. 
Fig. 4 - Una valutazione sintetica dei siti internet delle aziende europee analizzate
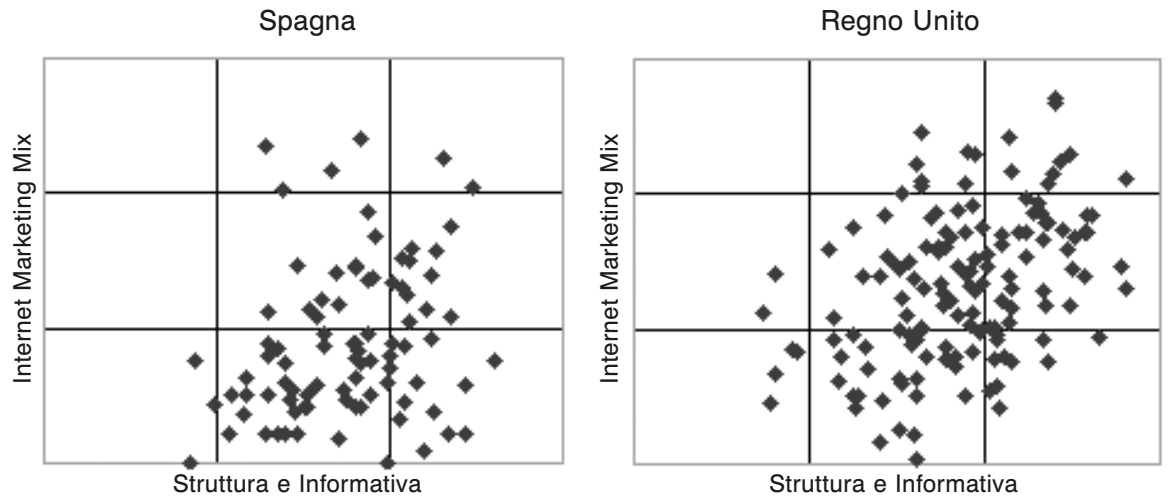

Germania

Francia
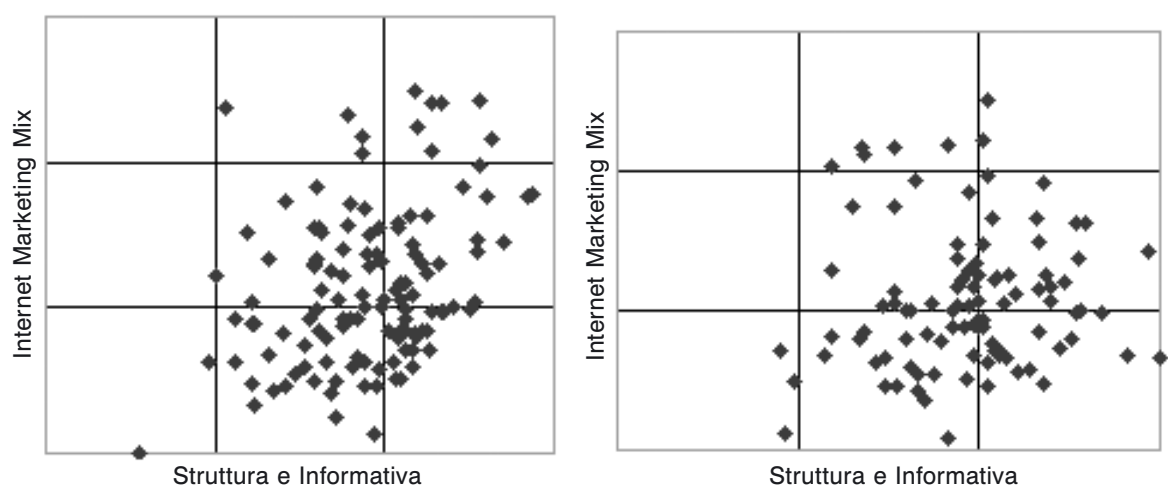

Struttura e Informativa

Italia

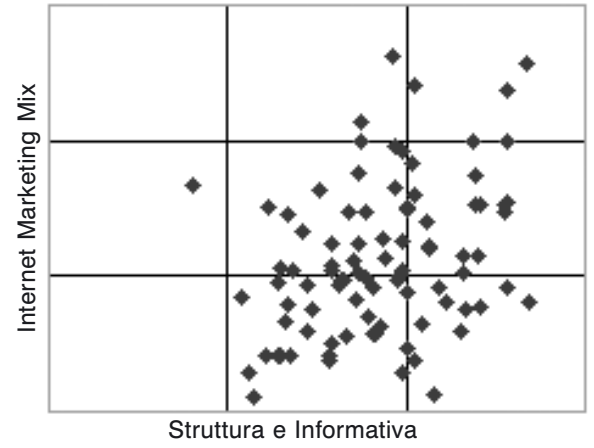

Il confronto tra le dispersioni relative ai singoli paesi mostra approcci dissimili. Tale considerazione risulta evidente verificando il posizionamento delle singole aziende nei nove quadranti previsti in ciascun grafico/paese. Anzitutto si nota un miglioramento dell'utilizzo di internet come leva di 


\section{E. Cedrola}

marketing da parte delle imprese italiane, anche se solo a distanza di un anno. Questo risultato è quasi esclusivamente attribuibile ai progressi raggiunti dalle imprese appartenenti al settore del turismo e della ristorazione, con qualche timida presenza da parte di imprese del settore dell'elettronica. Tale evidenza empirica conferma l'influenza sullo sfruttamento di internet da parte delle caratteristiche dell'ambiente competitivo in cui l'impresa opera, della diffusione della tecnologia e della "confidenza" della clientela con la rete.

La distribuzione delle imprese spagnole, tedesche e francesi è similare, pur evidenziando un forte orientamento al marketing di numerosi siti aziendali, soprattutto appartenenti al settore turistico. Per Francia e Germania alcune aziende eccellenti appartengono, come in Italia, al settore dell'elettronica e degli elettrodomestici. In posizione differente si trovano invece le imprese inglesi, che sottolineano un sostanziale investimento nell'internet marketing. La numerosità delle imprese classificate nei quadranti superiori è decisamente elevata e mette in luce il grande numero di operatori che interpreta internet in un'ottica strategica di marketing. Interessante poi notare come non ci sia una prevalenza di imprese eccellenti nel settore turistico: tutti i settori sono equamente rappresentati. Questo è indice di una maggiore correttezza interpretativa di internet, ma potrebbe anche essere un primo segnale di maggiore orientamento strategico delle imprese stesse.

\section{Conclusioni}

L'indagine empirica realizzata evidenzia la sostanziale attualità dello scenario già rappresentato da Vescovi nel 1999 e relativo all'introduzione di internet nel marketing mix aziendale delle PMI italiane, anche se le evoluzioni riscontrate sono numerose.

Gli aspetti che si mantengono tuttora critici si riferiscono alla definizione di una chiara strategia di marketing e di internet marketing, all'attuazione di cambiamenti nell'organizzazione e al coinvolgimento dell'impresa nel progetto di internet marketing. Migliorano invece la comprensione dei nuovi paradigmi di marketing (strutturali e relazionali) e l'integrazione con gli strumenti e le attività di marketing tradizionale, anche se limitatamente alle leve prodotto e distribuzione.

È dunque chiaro come internet giochi ancora un ruolo circoscritto a supporto del business delle imprese sia a livello domestico che internazionale, anche se i trend rilevati evidenziano progressivi e costanti sforzi per rendere i siti aziendali sempre più aperti all'incontro con clienti domestici e oltre confine.

I segnali colti sono però troppo deboli per derivarne giudizi ottimistici. Infatti, in merito al tentativo effettuato di verificare la correlazione tra gli 
indici ponderati di bontà del sito internet delle PMI campione e alcuni indicatori di solidità aziendale (indice di indebitamento, indice di disponibilità, R.O.I., R.O.E., indice di solidità finanziaria, cash flow $)^{7}$, non sono stati individuati legami di alcun tipo (dunque né correlazione diretta, né inversa), anche se intuitivamente avremmo potuto evidenziare alcune dipendenze reciproche, pur su tematiche specifiche. Ad esempio un'impresa con un buon indice di solidità finanziaria dovrebbe avere una buona valutazione di orientamento al mercato del sito internet, o quantomeno presentare tra la documentazione in rete $\mathrm{i}$ dati di bilancio e informazioni sulla propria attività, ma questo non avviene. Ci troviamo indubbiamente di fronte ad opportunità non sfruttate, soprattutto in tema di visibilità sui mercati internazionali.

Obiettivo futuro della ricerca sarà quello di approfondire l'analisi dei dati relativi al campione di imprese europee, oltre a quello di affinare le analisi di correlazione tra comportamenti in rete e indici di valutazione d'azienda, in modo da poter proporre risultati solidi sulla tematica in questione.

\section{Riferimenti bibliografici}

Amor D. (2000). E-business (r)evolution. Vivere e lavorare in un mondo interconnesso. Milano: Tecniche Nuove.

Ancarani F. (2002). Il pricing nell'economia digitale. Milano: Etas.

Blattberg R.C. and Deighton J. (1991). Interactive marketing: exploiting the age of addressability. Sloan Management Review, 33, 1:5-14.

Butera F. (2001). Il campanile e la rete. Milano: Il Sole 24 Ore.

Canavan O., Henchion M. and O'Reilly S. (2007). The use of internet as a marketing channel for Irish specialty food. International Journal of Retail \& Distribution Management, 35, 2:178-195.

Castellett M. (2007). Marketing di relazione. Strategie d'impresa, politiche di gestione della complessità, rete e management. Milano: Il Sole 24 ore.

Cedrola E. (2002 a). L'impatto di internet sull'attività degli intermediari finanziari: il caso delle banche in Rete. Milano:Quaderni di ricerca del Dipartimento di Scienze dell'Economia e della Gestione Aziendale, ISU Università Cattolica.

Cedrola E. (2002 b). Les nouveaux services bancaires liés à internet. Comparaison entre l'Italie, l'Europe et les Etats-Unis. Revue Française du Marketing, 189/190:105-118.

Cedrola E. (2003). Servizi di pubblica utilità ed internet: un focus sul settore postale europeo. Atti del Congresso Internazionale Marketing Trends, Venezia 28-29 novembre.

Cedrola E. (2004). internet e i servizi di pubblica utilità: i trasporti ferroviari in Europa. In: Mele R. e Parente R. e Popoli P., a cura di, I processi di deregolamentazione dei servizi pubblici. Vincoli, opportunità istituzionali e condizioni operative. Rimini: Maggioli Editore.

7. Di cui è stata fatta menzione solo nella nota 5 . 


\section{E. Cedrola}

Cedrola E. (2005). Il marketing internazionale per le piccole e medie imprese. Milano: McGraw-Hill.

Chaffey D., Mayer R., Johnston K. e Ellis-Chadwick F. (2001). Internet marketing. Milano: Apogeo.

Cioppi M. (2006). Il sito web aziendale nelle PMI: potenzialità elevate e realtà moderate. Piccola Impresa/Small Business. 1:147-175.

Corbae G., Jensen J.B. and Dirk Schneider (2003) Marketing 2.0 - Strategies for Closer Customer Relationships, Milano: Springer.

Dholokia R.R., Kshetri N. (2004). Factors impacting the adoption of the Internet among SMEs. Small Business Economics, 23:311-322.

Gilmore A., Gallagher D. and Henry S. (2007). E-marketing and SMEs: operational lessons for the future. European Business Review, 19, 3:234-247.

Hoffman D.L. and Novak T.P. (1996). Marketing in hypermedia computer-mediated environments: conceptual foundations. Journal of Marketing, 60, July:50-68.

Ioppolo D. e Scazzoso S. (1999). Analisi delle prime applicazioni di internet, in Scott W.G., Murtula M., a cura di, Il commercio elettronico. Verso nuovi rapporti tra imprese e mercati, Torino: Isedi Utet.

Kai-Uwe Brock J. (2000). Information and technology in the small firms, in Carter S., in Dylan-Jones D. (Eds), Enterprise and the Small Business, Financial Times Prentice-Hall.

Kaynak E., Tatoglu E. and Kula V. (2005). An analysis of the factors affecting the adoption of electronic commerce by SMEs. International Marketing Review, 22,6:623-640.

Kotler P. (2004). Il marketing che cambia. Milano:Il Sole 24 ore.

MacGregor R. and Vrazalic L. (2007). E-commerce in Regional Small do Medium Enterprises. New York, Hershey: IGI Publishing.

MacGregor R., Vrazalic L., Carlsson S., Bunker D. and Magnusson M. (2002). The impact of business size and business type on small business investment in electronic commerce: a study on Swedish small businesses. Australasian Journal of Information Systems, 9, 2:31-39.

Martin L.M. and Matlay H. (2001). "Blanket" approaches to promoting ICT in small firms: some lessons from the DTI ladder adoption model in the UK. internet Research: Electronic Networking Applications and Policy, 11, 5:399-410.

Naisbitt J. (1982). Megatrends: Ten New Directions Transforming Our Lives. New York:Warner Books.

Namiki N. (1988). Export strategy for small business. Journal of Small Business Management, 26, April:32-37.

Pionassi F. (2004). Comunità virtuali e piccole medie imprese. Forme alternative di presenza in rete, Milano:Franco Angeli.

Poon S. and Swatman P.M.C. (1997). Small Business use of internet. International Marketing Review, 14, 3:385-402.

Prandelli E. and Verona G. (2002). Marketing in rete. Milano:McGraw-Hill.

Prendelli E. and Verona G. (2006). Marketing in rete: oltre internet verso il nuovo marketing. Milano: McGraw-Hill.

Sadowski B.M., Maitland C., Van Dongen J. (2002). Strategic use of the Internet by small.and medium-sized companies: an esploratory study. Information Economics and Policy, 14:75-93.

Stockdale R. and Standing C. (2004). Benefits and barriers of electronic marketplace participation: an SME perspective. Journal of Enterprise Information Management, 17, 4:301-311. 
Stockdale R. and Standing C. (2006). A classification model to support SME e-commerce adoption initiatives. Journal of Small Business and Enterprise Development, 13, 3:381-394.

Stone M. (2003). SME e-business and supplier-customer relations. Journal of Small Business and Enterprise Development. 10, 3:345-353.

Veragouth P. (2000). Il marketing on-line e i suoi strumenti operativi. Strategie e tecniche per l'e-business. Brescia:Contactum Italia www.ilmarketingonline.com. (ultimoaccesso 19/08/2008).

Verhoest P. and Hawkins R. (2001). Impatti e prospettive del commercio elettronico. Lettera Marketing ABI, 2:28-34.

Vescovi T. (1999). internet, marketing e imprese minori: quali problemi? Micro \& Macro Marketing, VIII, 2:247-263.

Vescovi T. (2007). Il marketing e la rete. La gestione integrate del web nel business. Comunicazione, e-commerce, sales management, business to business. Milano: Il Sole 24 Ore.

Yadav M.S. and Varadarajan P.R. (2005). Understanding product migration to the electronic marketplace: a conceptual framework. Journal of Retailing, 81, 2:125140.

Zeff R. and Aronson B. (1999). Advertising on the internet. New York: John Wiley \& Sons. 